\title{
Potential of a Compton camera for high performance scintimammography
}

\author{
Lisha Zhang ${ }^{1}$, W Leslie Rogers ${ }^{2}$ and Neal H Clinthorne ${ }^{2}$ \\ ${ }^{1}$ Department of Biomedical Engineering, University of Michigan, Ann Arbor, MI 48109, USA \\ 2 Department of Radiology, University of Michigan, Ann Arbor, MI 48109, USA \\ E-mail: lishaz@umich.edu
}

Received 30 June 2003, in final form 26 November 2003

Published 30 January 2004

Online at stacks.iop.org/PMB/49/617 (DOI: 10.1088/0031-9155/49/4/011)

\begin{abstract}
In this paper, we present a novel approach to scintimammography that is based on the Compton camera principle. We analyse the performance of our scheme using Monte Carlo simulations. In particular, we evaluate the detection efficiency, spatial resolution and lesion visibility of the system at several gamma photon energies. The simulation results show that the proposed technique achieves an absolute detection efficiency of 0.03 and a full width at half maximum resolution of $3.8 \mathrm{~mm}$ at $141 \mathrm{keV}$ photon energy for point sources $2.5 \mathrm{~cm}$ deep in a $5 \mathrm{~cm}$ thick breast phantom using $5 \mathrm{~mm}$ thick silicon detectors. Furthermore, our approach shows good performance in lesion detection, especially at high gamma photon energies, where mechanically collimated systems perform poorly due to severe septal penetration. With total collected counts of 1.35 million, equivalent to a $30 \mathrm{~s}$ acquisition time for an activity concentration level of $3.7 \mathrm{kBq} \mathrm{ml}^{-1}\left(100 \mathrm{nCi} \mathrm{cm}^{-3}\right)$ in normal breast tissue, and a tumour-to-background ratio of $8: 1$, our system can clearly reveal an $8 \mathrm{~mm}$ diameter tumour that is located $2.5 \mathrm{~cm}$ deep in a $500 \mathrm{ml}$ breast phantom. We also present a simulation-based quantitative performance comparison between the proposed scintimammographic system and the compact collimated scintimammographic system in the task of lesion detection under a clinical imaging situation using a non-prewhitening matched filter observer model. Our comparison demonstrates that for the same imaging time, the two systems have a comparable performance in detecting an $8 \mathrm{~mm}$ tumour at $141 \mathrm{keV}$, with the proposed system performing marginally better. However, the proposed scintimammographic system clearly outperforms the compact collimated counterpart in the detection of a $5 \mathrm{~mm}$ tumour. We also investigate the contribution of scatter and direct radiation from adjacent organs. We find that the background contribution of liver to the right breast is $30 \%$ at $141 \mathrm{keV}$, which can be reduced to $4.8 \%$ with shielding.
\end{abstract}




\section{Introduction}

Excluding skin cancer, breast cancer is the most common malignancy, and the second leading cause of cancer death among women in the United States. It is estimated that approximately 211300 American women were expected to be diagnosed with breast cancer and 39800 would die from it in 2003 (Ries et al 2003). Despite its high incidence, patients with breast cancer generally have good prognosis, with a 10 -year survival rate of nearly $96 \%$ if detected at an early stage (less than $2 \mathrm{~cm}$ in size).

Currently, mammography remains the only validated method for breast cancer screening. It has very high spatial resolution and a sensitivity of $85 \%-90 \%$. Of the newly diagnosed cancers by mammographic screening, nearly $40 \%$ are ductal carcinoma in situ (DCIS) compared to 5\% prior to the use of mammography (Ernster et al 1996, Pandya et al 1998). The wide use of mammographic screening and timely treatment have led to a recent decline in breast cancer mortality in the United States (Blackman et al 1999).

Despite its successes, mammography is not free of limitation. It has a low specificity and positive predictive value, ranging from $50 \%$ to $60 \%$ and $10 \%$ to $40 \%$, respectively. In particular, it is not reliable for detecting tumours in patients with dense, altered or implanted breasts (Cutrone et al 1999, Jackson et al 1993). In radiographically dense breasts, the sensitivity of mammography decreases to 68\% (Rosenberg et al 1998). Several alternative imaging modalities have been investigated for their roles in improving breast cancer detection. None of the established technologies, however, is superior to mammography so far. They can only serve as an adjunct to mammography in the diagnosis and management of breast cancer.

The widely studied imaging techniques include ultrasound, magnetic resonance imaging (MRI) and scintimammography. Ultrasound has proved valuable in evaluating patients with dense breasts and differentiating cysts from solid masses. It has proved to be useful for the evaluation of ruptured silicone breast implants. However, it cannot differentiate the malignant and benign lesions very well, and it is also extremely operator dependent. MRI has superb spatial resolution, which makes it a very sensitive test. The reported sensitivity of MRI for detecting invasive breast cancer has approached 100\% (Orel et al 1994). It is also useful for evaluating the actual size of a tumour and identifying additional foci of breast cancer that are not evident in mammography. However, its low specificity and high cost limit its use in breast imaging. Scintimammography, a technique that identifies physiological properties instead of morphologic changes, has shown promise in identifying and locating primary breast tumours. Unlike mammography, it is independent of breast radio-density, which makes it particularly useful in patients with radiographically dense breasts (Khalkhali et al 1995). It is also valuable in monitoring recurrent cancer and tumour response to neoadjuvant chemotherapy for locally advanced breast cancer. Studies have shown that rapid tumour clearance of ${ }^{99 \mathrm{~m}} \mathrm{Tc}-$ sestamibi may indicate lack of response to chemotherapy (Ciarmiello et al 1998). Another important application of scintimammography is assessment of multifocal disease, which has proved to be useful for employing different surgical approaches such as lumpectomy with adjuvant radiation therapy or mastectomy. However, the utility of scintimammography is limited by its low sensitivity for tumours less than $1 \mathrm{~cm}$. To detect tumours of small size, an instrument with very high spatial resolution and detection efficiency is required. Unfortunately, a conventional general purpose gamma camera has only mediocre spatial resolution and poor counting efficiency. To overcome this problem, researchers have made substantial efforts to develop compact, high spatial resolution gamma cameras dedicated to scintimammography (Kim et al 2000, Levin et al 1997, Pani et al 1996, 1998, Pieper et al 2001, Williams et al 2000, Wojcik et al 1998). Most of those systems are based on position sensitive photomultiplier tubes 
of various sizes coupled to pixellated or continuous YAP:Ce, $\mathrm{CsI}(\mathrm{Na}), \mathrm{CsI}(\mathrm{Tl})$ and $\mathrm{NaI}(\mathrm{Tl})$ scintillation detectors. The reported systems can achieve an intrinsic spatial resolution of $2 \mathrm{~mm}$ or less, and an energy resolution ranging from $12 \%$ to $37 \%$ at $141 \mathrm{keV}$.

These dedicated breast imagers have superb spatial resolution. However, they still suffer from low efficiency, as do their conventional counterparts, due to the mechanical collimation, although a limited gain in detection efficiency is possible by trading off the achievable spatial resolution. As an alternative to the above scintimammographic imagers, significant progress has been made in the development of a breast-specific positron emission mammographic (PEM) instrument (Thompson et al 1994, Murthy et al 2000). This compact positron emission tomography (PET) imager, designed specifically for breast imaging, has an intrinsic spatial resolution of $2.8 \mathrm{~mm}$, a counting efficiency of $3 \%$ and a coincidence resolving time of $12 \mathrm{~ns}$. Although it achieves much improved efficiency in the centre of field of view (FOV) over the dedicated gamma camera, PEM suffers from a substantially decreased efficiency towards the edge of detectors, causing reduced sensitivity in identifying lesions close to the chest wall, a situation currently of great interest. Whereas, the detection efficiency of Compton camera based scintimammography decreases slowly with respect to position, and it can image well beyond the edge of the detector.

In this paper, we present a new approach to scintimammography that is based on the Compton camera principle and is potentially useful for detecting breast lesions less than $1 \mathrm{~cm}$. Although extensive investigations have been conducted by our group in assessing the value of Compton camera for general purpose single photon emission computed tomography (SPECT) relative to conventional instruments, the concept of a Compton camera based scintimammographic system is new and has not been studied. In this work, we investigate the performance, such as detection efficiency and spatial resolution, of the proposed system. In addition, we evaluate background radiation due to non-breast activity and its impact on the reconstructed images. We also quantitatively evaluate and compare the performance of the proposed system and a state-of-the-art dedicated mechanically collimated scintimammographic system for detecting lesions of various sizes, tumour-to-background $(\mathrm{T} / \mathrm{B})$ ratios and depths in a clinical imaging situation.

\section{Materials and methods}

\subsection{Description of the system}

The proposed Compton camera based scintimammographic imaging system comprises position and energy sensitive scatter and absorption detectors. The scatter and absorption detectors detect the recoil electron and scattered photon in time coincidence. Based on the energy deposition and position information in both detectors, the origin of the photon can be determined within a conic ambiguity. Spatial resolution is typically limited by the precision in determining the scattering angle, which in turn is primarily limited by Doppler broadening and energy resolution of the scatter detector. Silicon is chosen as the scatter detector material due to its high Compton-to-total cross-section ratio, excellent energy resolution, room temperature operation and small Doppler broadening compared to germanium $(\mathrm{Ge})$ and cadmium zinc telluride (CZT). Sodium iodide (NaI) is chosen as the absorption detector for its good energy resolution, availability in large quantities and cost-effectiveness.

Figure 1 shows the schematic diagram and patient scan setup of the proposed scintimammographic system. The system consists of two pixellated silicon detectors and a dual-head gamma camera. Unlike a conventional dual-head gamma camera, the imaging heads use no collimators, but are operated in time coincidence with the adjacent silicon detector 


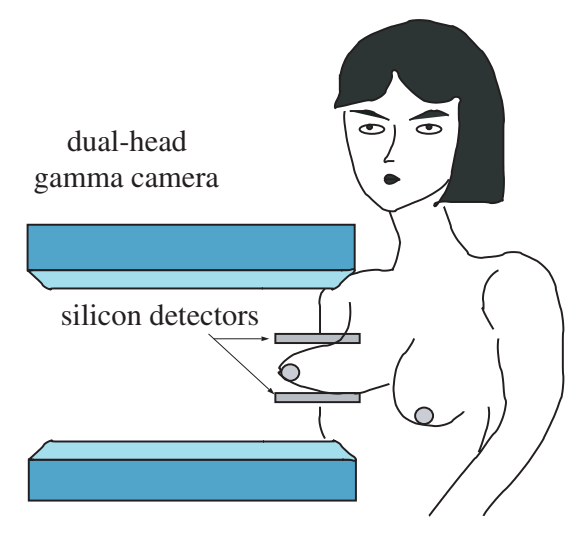

Figure 1. System diagram.

to detect the energy and position of photons scattered from the silicon detector. Each silicon detector is composed of a stack of $1 \mathrm{~mm}$ thick $10 \mathrm{~cm} \times 10 \mathrm{~cm}$ pixellated silicon detectors. The total thickness varies from $1-10 \mathrm{~mm}$, but the voxel size is fixed at $1 \mathrm{~mm}^{3}$. This ensures adequate coverage of an average-sized single breast in the FOV of the detector. The optimal choice of silicon detector thickness is determined using Monte Carlo simulations based on performance and cost considerations. The dual-head gamma camera is composed of two $40 \mathrm{~cm}$ wide, $40 \mathrm{~cm}$ deep and $2 \mathrm{~cm}$ thick NaI scintillation detectors with a $3 \mathrm{~mm}$ full width at half maximum (FWHM) intrinsic spatial resolution. The silicon detectors are placed right on the top and bottom of the target breast. The separation of two silicon detectors is $5 \mathrm{~cm}$, which is an average thickness for mildly compressed breast. The two heads of the gamma camera are placed parallel to the silicon detectors, but offset to clear the chest wall. The shortest distance between each camera head to its nearest silicon detector is set to $5 \mathrm{~cm}$. The small separation between the silicon detector and the NaI detector is preferred to increase the solid angle subtended by the absorption detector. The patient is in a seated position to allow craniocaudal views to be imaged conveniently. The craniocaudal view is chosen in our study for easy comparison with the mammography images.

The proposed Compton camera based scintimammographic (C-SMM) system significantly improves the detection efficiency by eliminating the mechanical collimator. Furthermore, by placing silicon detectors directly on the target, the solid angle subtended by silicon detectors is also increased. Both lead to greatly improved sensitivity over conventional scintimammography. The closer distance between the silicon detector and breast also improves spatial resolution for a given angular uncertainty. In addition, the proposed C-SMM geometry allows better access to the area close to the chest wall and the medial aspect of the breast.

\subsection{Phantoms}

Two $5 \mathrm{~cm} \times 10 \mathrm{~cm} \times 10 \mathrm{~cm}$ slabs of soft tissue, with a thickness of $5 \mathrm{~cm}$, are used to simulate the mildly compressed breasts. They are attached to the Zubal phantom (Zubal and Harrell 1992) to form an anatomically correct anthropomorphic phantom for the simulation. This allows accurate modelling of scattering and attenuation in a realistic imaging environment. The effect of various thicknesses of compressed breasts on the image quality has been previously studied (Pani et al 1997). In this paper, we are not trying to evaluate the image quality quantitatively due to varying amounts of breast compression. Instead, we fix the thickness of the compressed breast to be $5 \mathrm{~cm}$, a typical thickness for the mildly compressed breast. 


\subsection{Simulation}

For completeness, we briefly describe the implementation of our Monte Carlo simulation (Zhang et al 2000). The original Zubal phantom has a total of $128 \times 128 \times 243$ voxels with $4 \mathrm{~mm}$ isotropic resolution. Considering the geometry and target to be imaged in our scintimammographic system, scattering due to the activity in the head and lower body (legs) is extremely low. Therefore, the Zubal phantom was cropped along the axial direction to exclude the legs and head. This reduces the memory usage and computation time with little compromise of simulation accuracy. Since the outer phantom voxels are simply composed of air, the phantom was further reduced in the transverse direction to eliminate unnecessary air voxels. The modified phantom has a total of $60 \times 100 \times 72$ voxels with $4 \mathrm{~mm}$ isotropic resolution. Each voxel was indexed to an internal organ with cross-section data generated based on the organ's composition and density given in ICRU's annual report (ICRU 1989). For organs whose composition is unavailable, soft tissue was used. We treat each individual voxel as a distinct region in spite of the homogeneity within each organ in order to easily track boundary crossing. The number of radioactive decays from each voxel was proportional to the corresponding activity concentration to simulate the desired source distribution in the phantom. A rectilinear array with the same size as the phantom was set up to hold the digital phantom. An input data file containing an organ index number for each voxel was sequentially read into the EGS4 user code, and each voxel was assigned to a medium based on this value. All the voxels in the phantom have the same transport cutoff energy. In the simulation, the photon was tracked down to $1 \mathrm{keV}$ and electrons were absorbed locally. Two slabs used to simulate the breasts were specified by their location, size and the direction of the normal vector of each plane forming the slabs. As previously mentioned, two silicon detectors were located on the top and bottom of the simulated breasts and the dual-head $\mathrm{NaI}(\mathrm{Tl})$ gamma camera was positioned so as to clear the chest wall. A sphere that includes both phantom and detectors was used to confine the photon tracking space. Photons are tracked as long as they are inside the sphere, which accurately models the scattering effects. To allow flexible use of developed Monte Carlo codes in various applications, any components other than the digital phantom, are mathematically described in continuous space independent of the finite resolution of the digital phantom, making it possible to model the small lesions embedded in the phantom accurately.

A good random number generator (RNG) is important for producing realistic results in Monte Carlo simulations. In our studies, a universal lagged-Fibonacci pseudo random number generator developed by Marsaglia (Marsaglia et al 1990) was used instead of a linear congruential random number generator. This RNG is machine independent and will generate identical sequences on all machines that support single-precision real numbers with 24 bit fractional parts (Bielajew and Walker 1993). In addition, the length of this random number generator is $2^{144}$ (about $2 \times 10^{43}$ ). It virtually guarantees that no simulation is duplicated.

EGS4 Monte Carlo simulations were conducted to investigate the performance of the proposed system. Specifically, we focused on its detection efficiency, spatial resolution and lesion visibility over a wide range of gamma photon energies. The effect of background from non-breast uptake was also studied. In all the simulations, the major factors that limit system performance were modelled including scattering (Compton and Rayleigh scattering), attenuation, Doppler broadening and spatial and energy resolutions of both scatter and absorption detectors. The position measurement in the silicon detector was estimated as the centroid of the pad in which the interaction occurs. The use of thick NaI crystal results in a high fraction of multiple interactions, thus an energy weighted position sum of each individual interaction was used to calculate the final position in the NaI detectors. An energy resolution 
of $1 \mathrm{keV}$, achievable in the latest generation Compton camera detector, was used for the silicon detectors (Meier et al 2002, Studen et al 2003). The FWHM spatial and energy resolutions of the $\mathrm{NaI}$ detectors were $3 \mathrm{~mm}$ and $10 \%$ at each photopeak of simulated radionuclides, respectively. A $20 \%$ symmetric energy window for each photopeak was used to select valid events. Valid events are those that are detected by the scatter and absorption detectors in time coincidence, that are single Compton scattered in the silicon detector and that have total energy deposition within the preset energy window. The silicon detector thickness was set to $5 \mathrm{~mm}$ for all simulations except for the detection efficiency study. The dual-head gamma camera was simulated by two $40 \mathrm{~cm} \times 40 \mathrm{~cm} \times 2 \mathrm{~cm} \mathrm{NaI}$ detectors. To evaluate the performance of the proposed system at different photon energies, we simulated the photon energies of several widely used radionuclides: ${ }^{99 \mathrm{~m}} \mathrm{Tc}(141 \mathrm{keV}),{ }^{111} \mathrm{In}(171 \mathrm{keV}, 245 \mathrm{keV}),{ }^{131} \mathrm{I}(364 \mathrm{keV})$ and ${ }^{18} \mathrm{~F}(511 \mathrm{keV})$. Two imageable photopeaks of ${ }^{111} \mathrm{In}$ at $171 \mathrm{keV}(90 \%)$ and $245 \mathrm{keV}(94 \%)$ were simulated independently with a $20 \%$ symmetric energy window set over each photopeak.

2.3.1. Detection efficiency. Detection efficiency was estimated by placing an isotropic point source in the breast. The depth of the point source was varied from $0.5 \mathrm{~cm}$ to $2.5 \mathrm{~cm}$ with a granularity of $0.5 \mathrm{~cm}$. Simulation of the point source at deeper depths is unnecessary due to the symmetry of the system. In order to determine the optimal silicon detector thickness for our system, we performed simulations for detector thicknesses of $1 \mathrm{~mm}, 2 \mathrm{~mm}, 5 \mathrm{~mm}$ and $10 \mathrm{~mm}$, respectively. The choice of detector thickness is determined by its overall performance in terms of detection efficiency and cost. Detection efficiency was calculated as the ratio of valid events to the total number of photons emitted. At each point source depth and gamma photon energy, approximately 10 million photons were simulated to achieve the desired statistics.

2.3.2. Spatial resolution. Two point sources spaced $5 \mathrm{~mm}$ apart were placed at the centre of a series of equispaced horizontal planes in the breast. The depth and energy of the point sources were the same as those in the efficiency study. Coincidences between silicon and $\mathrm{NaI}$ detectors that are single Compton scattered in silicon detectors, and that have total energy deposition within the $20 \%$ energy window, were accepted as the valid events and recorded in list-mode. A total of ten realizations were generated at each gamma photon energy and depth, and about 10000 valid events were collected at each realization. The data were reconstructed using the penalized list-mode maximum likelihood expectation maximization (LMML-EM) method with a pixel size of $1 \mathrm{~mm}$. Horizontal and vertical profiles taken through the peak of the reconstructed images were fitted with a Gaussian function. These fitted profiles were averaged to yield the FWHM spatial resolution for a single realization. The final FWHM spatial resolution was determined by averaging the results over ten realizations.

2.3.3. Lesion visibility. Two different experiments were conducted to evaluate the performance of the proposed scintimammographic system for identifying lesions less than $1 \mathrm{~cm}$ under ideal and more realistic conditions.

In the first experiment, the breasts were filled with low activity. Three spherical tumours with various sizes and radiotracer activities were inserted at different locations in the breast. Despite the fact that ${ }^{99 \mathrm{~m}} \mathrm{Tc}$ labelled radiotracer is the only radio-pharmaceutical used in current clinical scintimammography practice, potential radiotracers such as ${ }^{111} \mathrm{In},{ }^{131} \mathrm{I}$, and ${ }^{18} \mathrm{~F}$ with higher photon energies were also investigated. At each photon energy, two $8 \mathrm{~mm}$ diameter tumours with tumour-to-background $(\mathrm{T} / \mathrm{B})$ ratios of $8: 1$ and 10:1 were placed in the medial plane of the breast $(2.5 \mathrm{~cm}$ deep $)$. A $5 \mathrm{~mm}$ diameter tumour with a T/B ratio of $25: 1$ was 


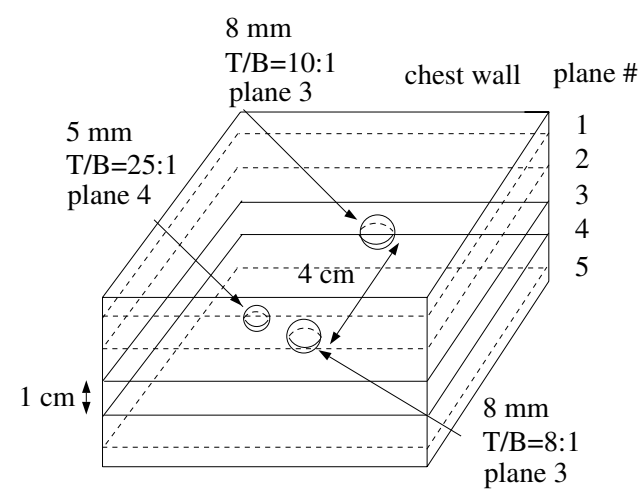

Figure 2. Diagram of simulated tumours in lesion visibility experiments. Planes delineated in solid line pass through the centres of tumours. Two $8 \mathrm{~mm}$ tumours with $\mathrm{T} / \mathrm{B}$ ratios of 8:1 and 10:1 are located in the medial plane, and a $5 \mathrm{~mm}$ tumour with a $\mathrm{T} / \mathrm{B}$ ratio of $25: 1$ is located in the plane $1 \mathrm{~cm}$ below the medial plane.

Table 1. Volumes of structures used in the phantom.

\begin{tabular}{ll}
\hline Structures & Volume $(\mathrm{ml})$ \\
\hline Left/right breast & 500 \\
Breast lesions & 0.065 and 0.268 \\
Heart & 598.7 \\
Liver & 1873.7 \\
\hline
\end{tabular}

located $1 \mathrm{~cm}$ below the medial plane. The schematic diagram of tumour sizes and locations within the breast phantom is shown in figure 2 . The $5 \mathrm{~mm}$ and $8 \mathrm{~mm}$ tumours were chosen in our study to represent the size of a pT1a and pT1b breast lesion in clinics, which are not reliably detected by the current scintimammography. The $\mathrm{T} / \mathrm{B}$ ratios assigned to $8 \mathrm{~mm}$ diameter tumours were 8:1 and 10:1 respectively, which are within the range of $\mathrm{T} / \mathrm{B}$ ratios observed in real ${ }^{99 \mathrm{~m}} \mathrm{Tc}$-sestamibi uptake in breast tumour (Maublant et al 1996). The tumours were positioned such that a deep region and a region close to the chest wall are included, where conventional scintimammography often performs poorly due to viewing position and large edge effects. In this experiment, the activity distribution was restricted to the breasts and lesions to represent the ideal imaging condition with no background radiation from non-breast sources. The setup, however, still allows scatter and attenuation caused by the complicated thorax structure to be modelled correctly.

The second experiment was similar to the first experiment, except that the activity was not confined to the breasts and lesions. Studies of human biodistribution of ${ }^{99 \mathrm{~m}} \mathrm{Tc}$ labelled methoxy-isobutyl-isonitrile (MIBI) show that there are high activities in the heart and liver (Savi et al 1989, Wackers et al 1989). Since the biodistribution of potential radiotracers at higher photon energies is unavailable and likely different from that of ${ }^{99 \mathrm{~m}} \mathrm{Tc}$-sestamibi, the activities in the breasts, heart and liver are arbitrarily approximated by concentration levels of 1:2:4 at all the gamma photon energies. The volumes of each structure filled with radioactivity are listed in table 1 . This experimental setup allows us to accurately model the background radiation due to the myocardium and liver activity, which has proved to be significant in conventional scintimammography.

Similar to the efficiency and spatial resolution studies, events with a single Compton scattering in silicon detector and total energy deposition in both silicon and $\mathrm{NaI}$ detectors within a $20 \%$ energy window were collected and recorded in list-mode. The imaging time 


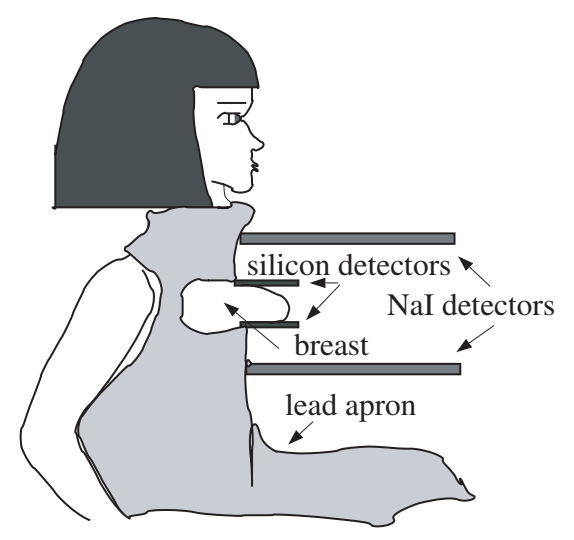

Figure 3. Patient scan setup with lead apron.

and amount of radioactivity in the breasts and lesions were constant in both experiments. Approximately 1.35 million counts were collected at each energy in the first experiment. The number of counts collected in the second experiment varied from 1.5 million to 2.0 million due to the variation in the fraction of background radiation at different photon energies. The collected data were then reconstructed using the penalized LMML-EM method. A total of five equispaced horizontal slices with $1 \mathrm{~cm}$ spacing as depicted in figure 2 were reconstructed simultaneously. Of the five reconstructed slices, two slices in the middle pass through the centre of the tumours. For these studies, a $2 \mathrm{~mm}$ pixel size was used instead of $1 \mathrm{~mm}$ in the spatial resolution study because of the increased computation required to reconstruct five planes.

2.3.4. Background radiation due to non-breast activity. It is well known that the image quality of conventional scintimammography is degraded by the background originating from high levels of non-specific activity in myocardium and liver. To quantify the background effect on the proposed C-SMM system, the detected direct and scattered counts from liver and heart were normalized to the counts from each breast and expressed as background counts to breast counts ratio. Again, radiotracers with energies ranging from $141 \mathrm{keV}$ to $511 \mathrm{keV}$ were simulated. The activity concentration ratio of breast to heart to liver was 1:2:4. A $20 \%$ symmetric energy window was placed on the energy sum of time-coincident events in the silicon and $\mathrm{NaI}$ detectors. Events falling within this window were collected, and the background counts to breast counts ratio was then calculated. To reduce background from non-breast uptake, a modified radiologic lead apron was used. The lead apron was similar to the standard radiologic lead apron except for a hole in front to allow the breasts to be imaged. For effective shielding at the higher energies, the thickness of lead is chosen as $0.32 \mathrm{~cm}$ (1/8 inch). Figure 3 shows the patient scan setup with shielding. To evaluate the effectiveness of shielding on the lesion visibility, we repeated the experiment described in the previous section, where the activity concentration ratio in breast, heart and liver was 1:2:4, using the same setup and imaging time, but with the lead apron. The collected valid events were reconstructed using the penalized LMML-EM method with a pixel size of $2 \mathrm{~mm}$.

\subsection{Image reconstruction}

In this work, the simulation data were recorded in list-mode and reconstructed as limited-angle tomograms using the penalized LMML-EM method. The seminal paper on the LMML-EM 
method has been proposed by Barrett et al (Barrett et al 1997, Parra and Barrett 1998), and its application to Compton camera reconstruction has been previously described in detail (Wilderman et al 1998, 2001). In this section, we briefly summarize the reconstruction method used in our study.

The LMML-EM algorithm proceeds in two steps: expectation step (E-step) and maximization step (M-step). In the E-step, the expectation of log likelihood of complete data given the measurement and current estimate of parameters is calculated as follows:

$$
\begin{aligned}
Q\left(\underline{\lambda} \mid \underline{\lambda}^{(n)}\right) & =E\left\{\ln f\left(\left\{X_{i j}\right\} \mid \underline{\lambda}\right) \mid Y_{i}, \underline{\lambda}^{(n)}\right\} \\
& =\sum_{i, j} \frac{Y_{i} a_{i j} \lambda_{j}^{(n)}}{\sum_{j} a_{i j} \lambda_{j}^{(n)}} \ln \left(a_{i j} \lambda_{j}\right)-\sum_{i, j} a_{i j} \lambda_{j}
\end{aligned}
$$

where $X_{i j}$ is the number of photons emitted from the $j$ th pixel and detected in the $i$ th measurement, $Y_{i}$ the $i$ th measurement that is 1 if the particle is detected in a given measurement and 0 otherwise, $a_{i j}$ the probability of detecting the gamma photon emitted from the $j$ th pixel in the $i$ th measurement, $\lambda_{j}$ the mean activity of the $j$ th pixel of the unknown object and $n$ is the iteration.

In the M-step, the maximum likelihood estimate is given by maximizing $Q\left(\underline{\lambda} \mid \underline{\lambda}^{(n)}\right)$ :

$$
\underline{\lambda}^{(n+1)}=\arg \max _{\underline{\lambda} \geqslant \underline{0}} Q\left(\underline{\lambda} \mid \underline{\lambda}^{(n)}\right) .
$$

This can be easily solved by differentiating equation (2) w.r.t. $\lambda_{j}$ and setting the differentiation to zero to yield the updated value $\underline{\lambda}^{(n+1)}$.

Maximizing $Q\left(\underline{\lambda} \mid \underline{\lambda}^{(n)}\right)$ generally leads to unacceptably noisy images because tomographic reconstruction is an ill-conditioned problem. To reduce the noise and produce more reasonable images, a quadratic penalty function was introduced. The penalty function we used has the form

$$
\alpha R(\underline{\lambda})=\alpha \sum_{j, k} r_{j k} \lambda_{j} \lambda_{k}
$$

where $\alpha$ is the regularization parameter and $r_{j k}$ is the weight assigned to neighbouring pixels of $\lambda_{j}$. A common choice of $r_{j k}$ could be $r_{j k}=1$ for four nearest neighbouring pixels and $r_{j k}=1 / \sqrt{2}$ for four diagonal neighbouring pixels and $r_{j k}=0$ otherwise.

With the penalty function added, the E-step remains the same, but the M-step becomes

$\underline{\lambda}^{(n+1)}=\arg \max _{\underline{\lambda} \geqslant \underline{0}} \phi\left(\underline{\lambda}^{\prime} \underline{\lambda}^{(n)}\right) \quad$ where $\quad \phi\left(\underline{\lambda} \mid \underline{\lambda}^{(n)}\right)=Q\left(\underline{\lambda}^{\prime} \underline{\lambda}^{(n)}\right)-\alpha R(\underline{\lambda})$.

Using the penalty function given in equation (4), the updated $\lambda_{j}^{n+1}$ is calculated as follows:

$$
\begin{aligned}
\lambda_{j}^{(n+1)}=-\frac{1}{2}( & \left.\frac{\sum_{k \neq j} r_{j k} \lambda_{k}}{r_{j j}}+\frac{\sum_{i} a_{i j}}{\alpha r_{j j}}\right) \\
& +\sqrt{\frac{1}{4}\left(\frac{\sum_{k \neq j} r_{j k} \lambda_{k}}{r_{j j}}+\frac{\sum_{i} a_{i j}}{\alpha r_{j j}}\right)^{2}+\frac{\lambda_{j}^{(n)}}{\alpha r_{j j}} \sum_{i} \frac{a_{i j} Y_{i}}{\sum_{j} a_{i j} \lambda_{j}^{(n)}}} .
\end{aligned}
$$

As the number of measurements increases, calculating and storing all the $a_{i j}$ becomes a daunting task. It can significantly slow down the computation. To solve this issue, a parallel version of the penalized LMML-EM algorithm was implemented using message-passing interface (MPI). The total number of counts was evenly distributed among $n$ processing nodes ( 1 root node and $n-1$ leaf nodes). Each node computes one partition of $a_{i j}$ and 
$\sum_{i \in I_{n}}\left(a_{i j} Y_{i}\right) /\left(\sum_{j} a_{i j} \lambda_{j}^{(n)}\right)$, where $I_{n}$ indicates a set of measurements assigned to the $n$th node. The calculated results were then sent back to the root node, where $\sum_{i} a_{i j}$ and $\sum_{i}\left(a_{i j} Y_{i}\right) /\left(\sum_{j} a_{i j} \lambda_{j}^{(n)}\right)$ were computed to update $\lambda_{j}^{(n+1)}$. Different from equation (6), we use a coordinate ascent method to update $\lambda_{j}^{(n+1)}$. The $\lambda_{j}^{(n+1)}$ is computed using the updated $\lambda_{k}^{(n+1)}$ instead of $\lambda_{k}^{(n)}$, where $k \in 0,1, \ldots, j-1$.

The intersection of the back-projected cone with each image plane is convolved with the sum of two Gaussian functions to approximate angular uncertainty resulting from the finite detector energy resolution and Doppler broadening. However, the width of the double Gaussian is fixed at a value suitable for the energy of ${ }^{99} \mathrm{~m} \mathrm{Tc}$ and not varied with either scattering angle or gamma energy. This leads to underestimated performance for high energy gamma rays.

Because of the silicon detector placement in these simulations, the object is incompletely sampled in angle. The resulting limited-angle tomograms are expected to have poorer resolution in depth. This spreads the tumour image data in the $z$ direction and decreases tumour image contrast. Furthermore, this can introduce image artefacts that depend upon the out of focus point response. Another problem occurs when the reconstructed image volume is smaller than the volume containing source activity, if some of this outside source activity is seen by the detector. This results in a survival probability that is not well defined (Zoglauer 2000). This also reduces image contrast because the out-of-volume activity is incorrectly reconstructed.

The image volume and the number of slices reconstructed are a compromise between attempting to obtain maximum depth resolution in the image and keeping image reconstruction time within realistic bounds. The choice of five planes appears to give acceptable depth resolution and reasonable contrast as demonstrated by the images.

\section{Performance comparison with compact collimated scintimammography}

\subsection{Modelling of a collimated scintimammographic system}

In this section, a state-of-the-art collimator-based compact, dedicated scintimammographic (SMM) system was modelled for performance comparison with the Compton camera based system in terms of lesion visibility and detectability. Since accurate modelling of a collimator is quite complicated and time consuming, we modelled an ideal parallel-hole collimator with no septal penetration and infinitesimal septal thickness. This simplification is fairly accurate at low gamma photon energies, due to the thin septal thickness and small fraction of septal penetration, which is no more than $2 \%$. The collimators used clinically have smaller open hole fraction and non-zero septal penetration, resulting in both reduced sensitivity and resolution. The resolution degradation due to septal penetration is especially problematic at high gamma photon energies. As a result, we expect that the actual collimator-based scintimammographic system will not perform as well as the results presented here.

The collimator modelled in our simulation is a modified version of low energy general purpose (LEGP) collimator (Nuclear Fields 2001). It has hexagonal holes with a $1.5 \mathrm{~mm}$ distance between opposite faces, zero septal thickness and $22 \mathrm{~mm}$ hole length. The sensitivity of the collimator is $14 \mathrm{cpm} \mathrm{kBq}^{-1}\left(518 \mathrm{cpm} \mu \mathrm{Ci}^{-1}\right)$ for ${ }^{99 \mathrm{~m}} \mathrm{Tc}$. For parallel-hole collimators, the sensitivity is approximately a constant, independent of the source position. The incoming photon that enters the front face of a collimator can pass through a collimator hole if it travels within a small angle with respect to the hole axis. This small angle is determined by the length and width of the collimator holes. 


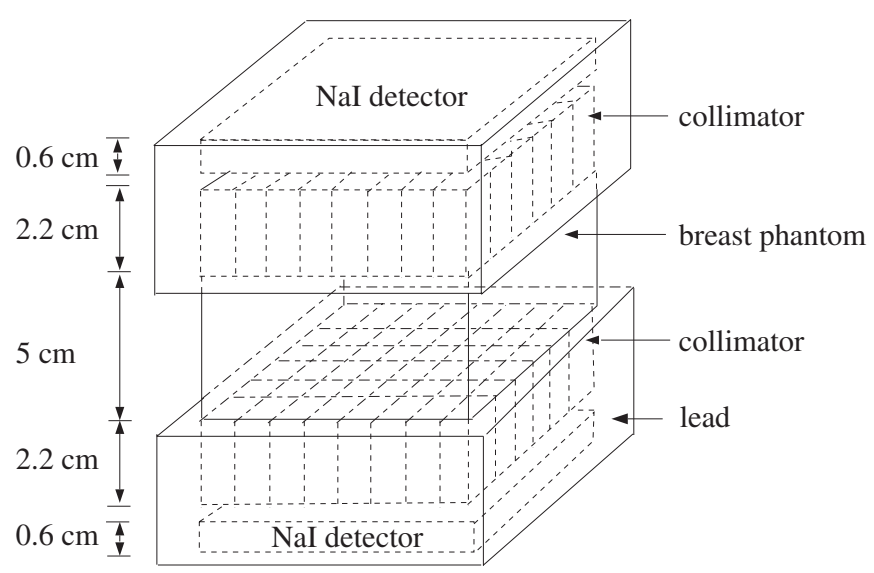

Figure 4. Diagram of the collimated, compact scintimammographic system.

The diagram of the collimated, compact scintimammographic system is shown in figure 4 . For illustration purposes, the square hole collimator was drawn instead of the hexagonal hole collimator. It consists of a pair of parallel-hole collimators, which are located on the top and bottom of the breast phantom. Two $10 \mathrm{~cm}$ wide, $10 \mathrm{~cm}$ deep and $0.6 \mathrm{~cm}$ thick NaI(Tl) detectors with a FWHM intrinsic spatial resolution of $2 \mathrm{~mm}$ and an energy resolution of $10 \%$ at $141 \mathrm{keV}$ are used to model the gamma camera dedicated to breast imaging (MacDonald et al 2000). The 10\% FWHM energy resolution was slightly better than that in MacDonald's study $(12.2 \%)$ to be consistent in our comparison of two systems. The separation between the $\mathrm{NaI}$ detector and the collimator is $0.2 \mathrm{~cm}$. The $\mathrm{NaI}(\mathrm{Tl})$ detectors are shielded all around with $0.95 \mathrm{~cm}(3 / 8$ inch) lead. A photon emitted from the source was tracked until it entered the front face of the collimator. Only those photons that could pass through the collimator hole were continuously tracked.

\subsection{Lesion visibility}

Two experiments conducted in section 2.3.3 for evaluating the performance of the C-SMM system in lesion visibility were repeated for the collimated system. The same phantom and activity concentration were used. Since the septal penetration is non-negligible at higher photon energies, meaningful evaluation was only conducted for ${ }^{99} \mathrm{~m}$ Tc labelled sestamibi. In the first experiment, activity was confined to the breasts. Two $8 \mathrm{~mm}$ diameter tumours with $\mathrm{T} / \mathrm{B}$ ratios of 8:1 and 10:1 were located in the medial plane of the breast. A $5 \mathrm{~mm}$ diameter tumour with a T/B ratio of 25:1 was located $1 \mathrm{~cm}$ below the medial plane. The positions of tumours were illustrated in figure 2 , the same as in the lesion visibility study in section 2.3.3. In the second experiment, the heart and liver were filled with the realistic activity levels common in clinical scintimammography studies. The relative activity concentration ratio for the normal breast, heart and liver was set to 1:3:12, the same as that of Majewski's study (Majewski et al 2001). In both experiments, the image acquisition time was the same as in the lesion visibility study of C-SMM for fair comparison, and the data were displayed as planar images with a pixel size of $1 \mathrm{~mm}$.

\subsection{Lesion detectability}

A signal known exactly (SKE) and background known exactly (BKE) lesion-imaging task was chosen to assess quantitatively the performance of the dedicated mechanically collimated 
SMM and C-SMM systems in lesion detection. Specifically, we employed a non-prewhitening matched filter (NPWMF) (Barrett et al 1993, ICRU 1996) as an observer. The NPWMF was used because of the easy computation of the test statistic. It is the optimum detector for a known signal in spatially uncorrelated or white noise, whereas the noise in the C-SMM and collimated SMM systems is correlated. The performance of NPWMF observer, therefore, is not optimal. Fortunately, the detection performance of human observer is compromised by the inability of the human visual system to undo spatial correlations. Nonetheless, the NPWMF is a reasonably good model to use because the significant source of variance in the reconstructed images arises from noise in the background distribution rather than from lesions in the low-contrast lesion detection task, which is our primary interest. The test statistic for NPWMF is given by

$$
\begin{aligned}
& \lambda_{\mathrm{NPW}}(\mathbf{g})=\left(E\left(\mathbf{g} \mid H_{1}\right)-E\left(\mathbf{g} \mid H_{0}\right)\right)^{t} \cdot \mathbf{g} \\
& \lambda_{\mathrm{NPW}}(\mathbf{g}) \geqslant \tau \quad \Rightarrow \quad H_{1}: \text { Tumour present } \\
& \lambda_{\mathrm{NPW}}(\mathbf{g})<\tau \quad \Rightarrow \quad H_{0}: \text { Tumour absent }
\end{aligned}
$$

where $H_{1}$ is the hypothesis of the tumour present, $H_{0}$ the hypothesis of the tumour absent, $E\left(\mathbf{g} \mid H_{1}\right)$ the mean reconstructed image under hypothesis $H_{1}, E\left(\mathbf{g} \mid H_{0}\right)$ the mean reconstructed image under hypothesis $H_{0}$ and $\tau$ the decision threshold.

The NPWMF observer simply performs inner product of the mean difference image and data under the test. Furthermore, the performance of each imaging system, under a reasonable assumption that the reconstructed images are approximately Gaussian distributed, can be completely specified by the signal-to-noise ratio (SNR) of the test statistic $\lambda_{\mathrm{NPW}}(\mathbf{g})$, which is shown as follows:

$$
\mathrm{SNR}^{2}=\frac{\left(E\left(\lambda(\mathbf{g}) \mid H_{1}\right)-E\left(\lambda(\mathbf{g}) \mid H_{0}\right)\right)^{2}}{\sigma^{2}(\lambda(\mathbf{g}))}
$$

where $E\left(\lambda(\mathbf{g}) \mid H_{1}\right)$ is the conditional mean of the test statistic under hypothesis $H_{1}, E\left(\lambda(\mathbf{g}) \mid H_{0}\right)$ the conditional mean of the test statistic under hypothesis $H_{0}$ and $\sigma^{2}(\lambda(\mathbf{g}))$ the variance due to the background distribution.

Using the SNR, the area under the ROC curve (AUC), which is a measure of detection performance, can be calculated as

$$
\mathrm{AUC}=0.5+0.5 \mathrm{erf}\left(\frac{\mathrm{SNR}}{2}\right) .
$$

By varying the tumour-to-background ratio, the SNR is calculated and the AUC can be correspondingly traced through the range of chosen T/B ratios. In the subsequent study, we limit our quantitative performance evaluation of the collimated SMM and C-SMM systems in detecting an $8 \mathrm{~mm}$ and a $5 \mathrm{~mm}$ tumour with the tracer of ${ }^{99 \mathrm{~m}} \mathrm{Tc}$-sestamibi. The tumour locations are the same as that described in the previous section, but of the two $8 \mathrm{~mm}$ tumours, only the tumour close to the chest wall was chosen. The activity concentration ratio in breast, heart and liver was 1:3:12.

The SNR estimates for both the collimated SMM and C-SMM systems were computed using multiple Monte Carlo realizations. For collimated SMM, projection data of 25 independent realizations of tumour-absent data were generated at $141 \mathrm{keV}$. For the C-SMM, a total of ten realizations were acquired with the same photon energy and activity distribution. Bootstrapping was used to generate 15 additional realizations of the C-SMM data. Both SMM and C-SMM realizations were then scaled to an equivalent imaging time of $10 \mathrm{~min}$ as in clinical scintimammography. For the C-SMM system, data were reconstructed using the parallel version of the penalized LMML-EM reconstruction algorithm on IBM SP2 supercomputers. 


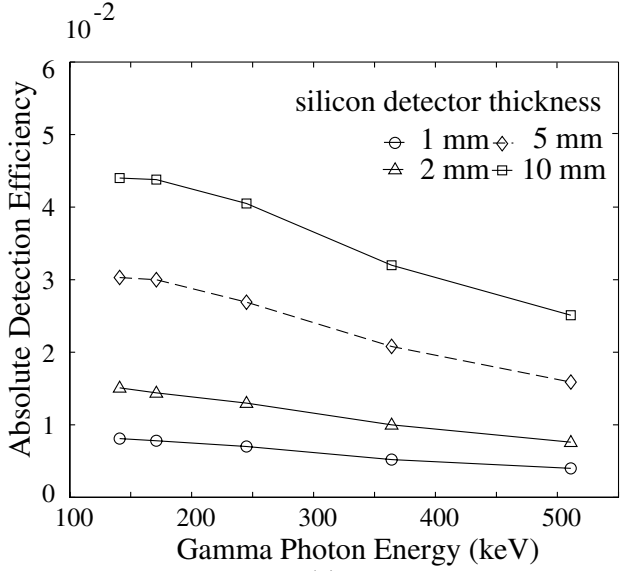

(a)

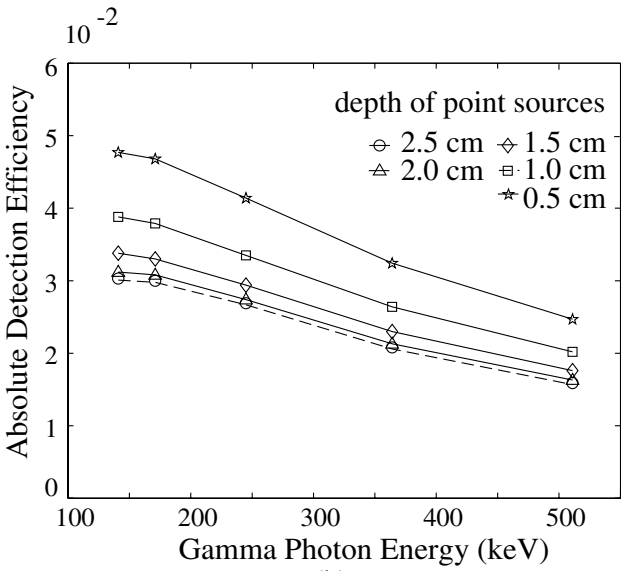

(b)

Figure 5. Absolute detection efficiency as a function of gamma photon energy, silicon detector thickness and the distance between the point source and the silicon detector for the C-SMM system. Effects of attenuation are included. (a) Point source located at the geometric centre of the medial plane $(2.5 \mathrm{~cm}$ deep in the breast phantom) and silicon detector thickness varied from $1 \mathrm{~mm}$ to $10 \mathrm{~mm}$. The dashed line shows the absolute detection efficiency at different gamma photon energies for a $5 \mathrm{~mm}$ thick silicon detector. (b) Silicon detector thickness fixed at $5 \mathrm{~mm}$ and point source located at the geometric centre of each equispaced horizontal plane through the breast in $0.5 \mathrm{~cm}$ intervals.

In contrast to the previous study in section 2.3.3, three instead of five equispaced slices in $1 \mathrm{~cm}$ intervals were reconstructed. The central slice is the mid-plane at a depth of $2.5 \mathrm{~cm}$. For the SMM system, the raw image is simply a projection image. However, for fair comparison, the raw image generated for the dedicated SMM system was convolved with a Gaussian kernel whose $\sigma$ is determined by the spatial resolution of the NaI detector and simulated collimator to reduce excessive noise. The difference image of the mean reconstructed images under two hypotheses forms a template, and can be approximated by the mean reconstructed image of lesion. A sequence of inner products of the template and multiple background realizations was used to estimate the variance term in the SNR formula. For the $8 \mathrm{~mm}$ and $5 \mathrm{~mm}$ tumours, the $\mathrm{T} / \mathrm{B}$ ratio is varied from $1: 1$ to $10: 1$, and $1: 1$ to $25: 1$, respectively. The AUC was then calculated at each $\mathrm{T} / \mathrm{B}$ ratio.

\section{Results and discussions}

\subsection{Efficiency}

Figure 5(a) shows the detection efficiency as a function of silicon detector thickness and photon energy. The point source was located at the geometric centre of the breast phantom. The efficiency is highest at $141 \mathrm{keV}$ for all silicon detector thicknesses, and decreases with increasing gamma photon energy due to reduced Compton-to-total cross-section ratio and increased detector penetration. The detection efficiency monotonically increases with the silicon detector thickness for a range of $1-10 \mathrm{~mm}$. In contrast to $10 \mathrm{~mm}$ thick silicon detectors, the detection efficiency for $5 \mathrm{~mm}$ thick silicon detectors is lowered by $35 \%$, but the number of electronic channels is reduced by $50 \%$, resulting in decreased system complexity and cost. Therefore, we choose $5 \mathrm{~mm}$ as the silicon detector thickness in all the subsequent simulations. Figure 5(b) shows the detection efficiency with respect to photon energy and distance from the 


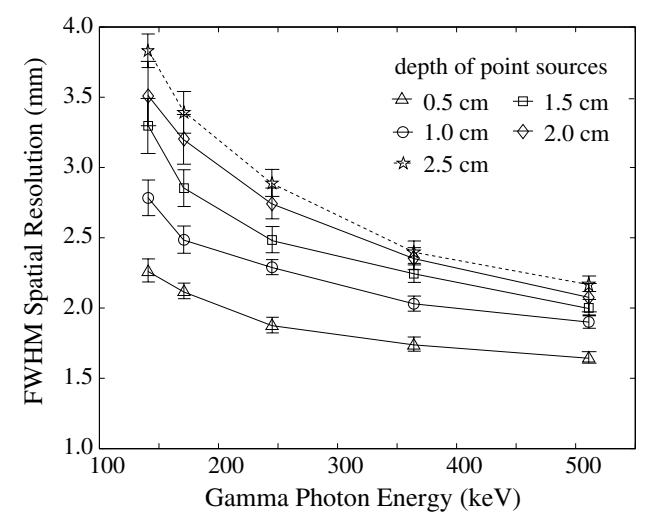

Figure 6. FWHM spatial resolution of the C-SMM system as a function of gamma photon energy and depth of point sources. Effects of attenuation are included. The dashed line shows the spatial resolution of the system with the point source located at the geometric centre of the medial plane ( $2.5 \mathrm{~cm}$ deep in the breast phantom). Error bars represent \pm 1 standard deviation of FWHM spatial resolution estimates.

point source to the silicon detector for a $5 \mathrm{~mm}$ thick silicon detector. The detection efficiency of the system decreases quickly with the increasing distance between the point source and the silicon detector due to the exponential law of attenuation and rapid decrease in the solid angle. The detection efficiency reaches the minimum with the source at the depth of $2.5 \mathrm{~cm}$ inside the phantom due to the symmetry of the system. The absolute detection efficiency of the system is 0.03 at $141 \mathrm{keV}$ with the point source located at a depth of $2.5 \mathrm{~cm}$, more than 2 orders of magnitude improvement over the dedicated mechanically collimated scintimammographic system, which has a detection efficiency of $2.1 \times 10^{-4}$ (McElroy et al 2002).

\subsection{Spatial resolution}

Figure 6 shows the system FWHM spatial resolution with the point source located at different depths in the breast phantom. The photon energies varied from $141 \mathrm{keV}$ to $511 \mathrm{keV}$ to include gamma ray emissions from ${ }^{99 \mathrm{~m}} \mathrm{Tc},{ }^{111} \mathrm{In},{ }^{131} \mathrm{I}$ and ${ }^{18} \mathrm{~F}$. The error bars delineate the \pm 1 standard deviation of the FWHM calculation. The spatial resolution of the proposed C-SMM system improves greatly at the higher photon energies primarily due to the reduced angular uncertainty related to Doppler broadening, but further improvement is marginal when the photon energy is higher than $364 \mathrm{keV}$. With the point source located at a depth of $2.5 \mathrm{~cm}$, the best spatial resolution was $2.2 \mathrm{~mm}$ FWHM, achieved at the energy of $511 \mathrm{keV}$. At $141 \mathrm{keV}$, the spatial resolution was $3.8 \mathrm{~mm}$ FWHM with the point source located medially $(2.5 \mathrm{~cm}$ deep in the breast phantom) and $2.25 \mathrm{~mm}$ when the depth of the point source was reduced to $0.5 \mathrm{~cm}$.

\subsection{Lesion visibility}

At each photon energy, a total of five limited-angle tomographic slices of breast images with a $10 \mathrm{~cm} \times 10 \mathrm{~cm}$ field of view were reconstructed simultaneously using the penalized LMML-EM method for the data collected in both experiments (activity restricted to breast, and activity in heart, liver and breast). These five slices are equally spaced at $1 \mathrm{~cm}$ intervals as shown in figure 2. Two planes delineated in solid line pass through the centres of tumours while the other three planes in dashed line define reconstructed planes that do not contain tumours. The reconstructed images are shown only for the right breast which has the most 


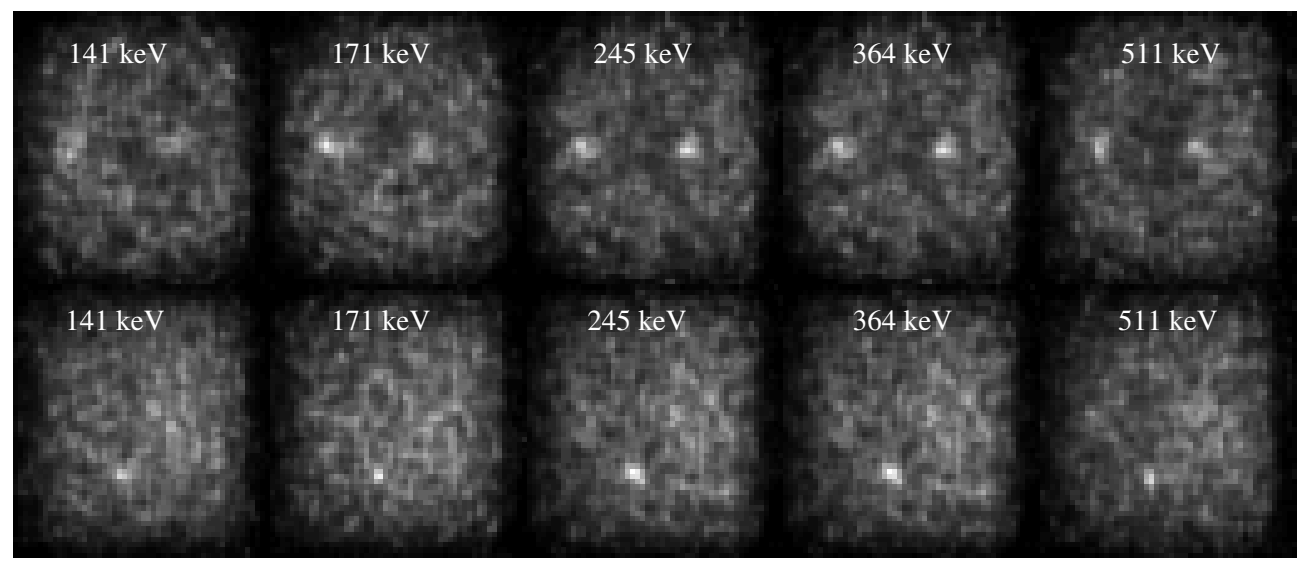

Figure 7. Penalized LMML-EM reconstruction of breast images from lesion visibility experiment 1 (only breasts and lesions contain activity). Top row: medial plane. Bottom row: $1 \mathrm{~cm}$ below the medial plane. Two $8 \mathrm{~mm}$ tumours with T/B ratios of 8:1 and 10:1 are located in the medial plane. A $5 \mathrm{~mm}$ diameter tumour with a T/B ratio of $25: 1$ is located in the plane $1 \mathrm{~cm}$ below the medial plane.

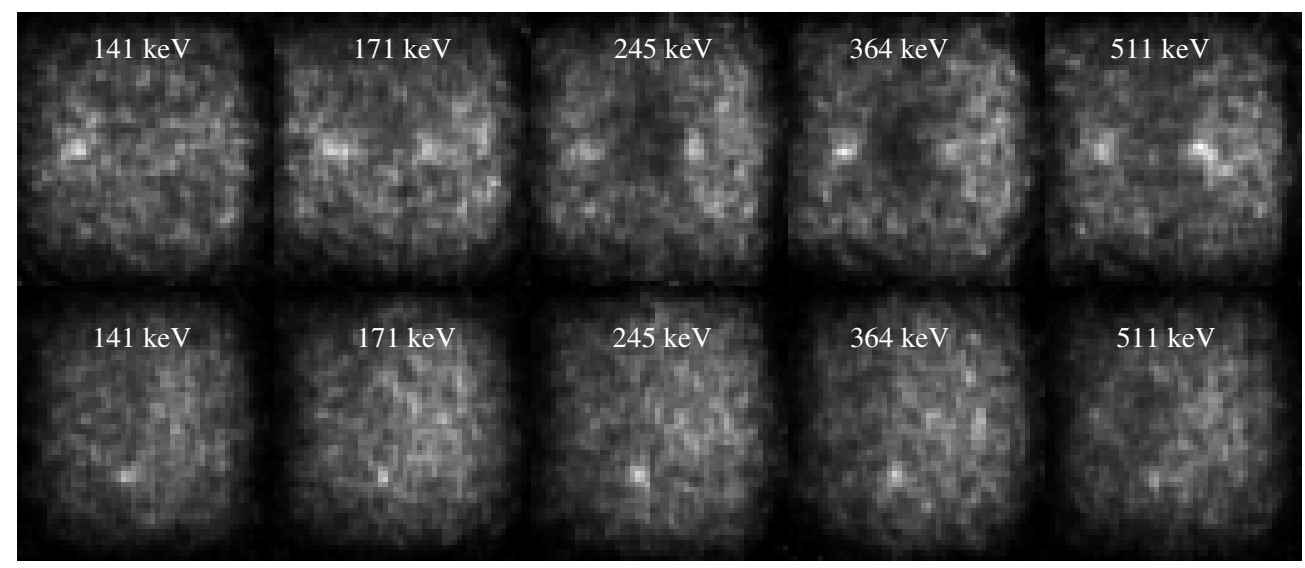

Figure 8. Penalized LMML-EM reconstruction of breast images from lesion visibility experiment 2 (activity concentration ratio among breast, heart and liver is 1:2:4). Top row: medial plane. Bottom row: $1 \mathrm{~cm}$ below the medial plane. Two $8 \mathrm{~mm}$ tumours with $\mathrm{T} / \mathrm{B}$ ratios of 8:1 and 10:1 are located in the medial plane. A $5 \mathrm{~mm}$ diameter tumour with a T/B ratio of 25:1 is located in the plane $1 \mathrm{~cm}$ below the medial plane.

background contamination due to significant liver uptake. Figure 7 shows the craniocaudal views of reconstructed images at the various energies with the activity restricted to the breasts only. Figure 8 shows the breast images with an activity concentration ratio of 1:2:4 in breast, heart and liver. In both figures, the top row of images corresponds to the medial slices and the bottom row of images corresponds to the slices $1 \mathrm{~cm}$ below the medial slices. In each image, the left side is closer to the chest wall. The size of each image is $52 \times 52$ with a pixel size of $2 \mathrm{~mm}$. Events with Compton scattering angle less than $30^{\circ}$ are rejected in the reconstruction.

The images without myocardium and liver activity are less noisy and have better contrast compared with the ones with activity in the heart and liver. Both $5 \mathrm{~mm}$ and $8 \mathrm{~mm}$ diameter lesions are clearly visible at all the energies, including the gamma ray emission 


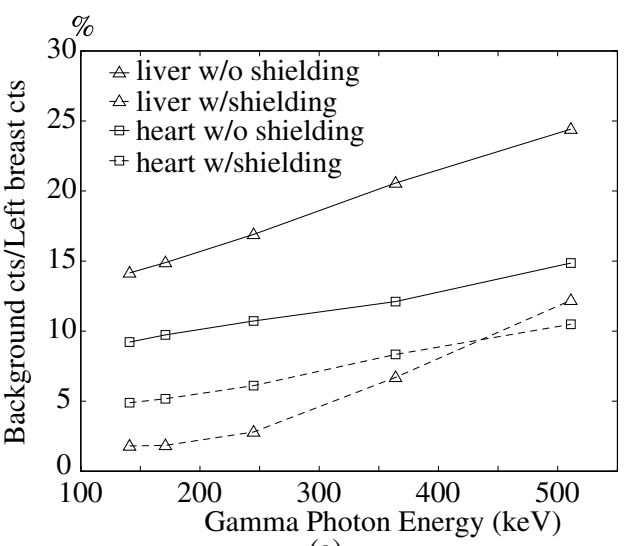

(a)

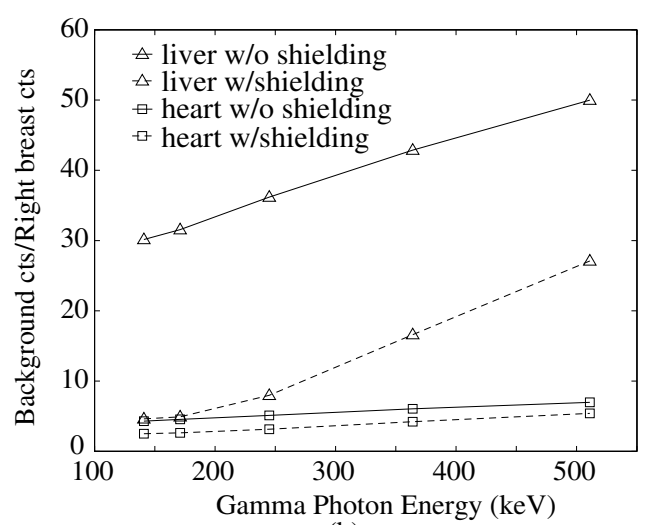

(b)

Figure 9. Non-breast background radiation with and without shielding: (a) left breast and (b) right breast.

from ${ }^{99 \mathrm{~m}} \mathrm{Tc}$-sestamibi. The improved performance at higher gamma photon energies is quite obvious with the best-contrast images obtained at the energies of $245 \mathrm{keV}$ and $364 \mathrm{keV}$. With increasing gamma photon energy, better spatial resolution was achieved, but detection efficiency decreased. Therefore, the best performance was achieved at medium energies, where the good spatial resolution and fairly high detection efficiency were simultaneously achieved. The images with myocardium and liver activity have reduced contrast between the lesions and normal background breast tissue. Due to the contamination from non-breast background radiation, the $8 \mathrm{~mm}$ lesion with a T/B ratio of $8: 1$ is not visible at the photon energy of $141 \mathrm{keV}$. The $5 \mathrm{~mm}$ lesion is less visible at the energy of $511 \mathrm{keV}$ due to the increased fraction of background radiation. With an approximate activity concentration level of $100 \mathrm{nCi} \mathrm{cm}^{-3}$ (Cinti et al 2001) in the normal breast tissue for clinical scintimammography study, the acquisition time for collecting 1.35 million counts from the breasts for our proposed system is only $30 \mathrm{~s}$ compared to 44 min using a dedicated mechanically collimated gamma camera. Of the 1.35 million collected counts, only 5750 come from the $8 \mathrm{~mm}$ lesion with a $\mathrm{T} / \mathrm{B}$ ratio of $8: 1$, compared to 1334890 counts from the normal breast. With a 10 min acquisition time, a typical imaging time for clinical scintimammography study, our system would acquire about 27 million counts, which undoubtedly ensures significant improvement of image quality. However, limited by the computation complexity of the reconstruction algorithm, only a fraction of the counts collected with realistic scan durations was used for image reconstruction in the lesion visibility study. Furthermore, the quality of the images is degraded by inconsistent data from background sources lying outside the reconstructed image volume. This can be reduced by reconstructing a larger field of view. Lesion visibility could be improved by increasing the range of angular sampling. This could be accomplished by redistributing the silicon detectors over a larger angular range. This may reduce sensitivity and will need additional modelling to determine if it improves performance.

\subsection{Background radiation due to non-breast activity}

Figures 9(a) and (b) illustrate the contribution of heart and liver background radiation to the left and right breasts, respectively. To demonstrate the effectiveness of simple shielding, the scattered and direct counts due to non-breast activity with lead apron shielding are also 


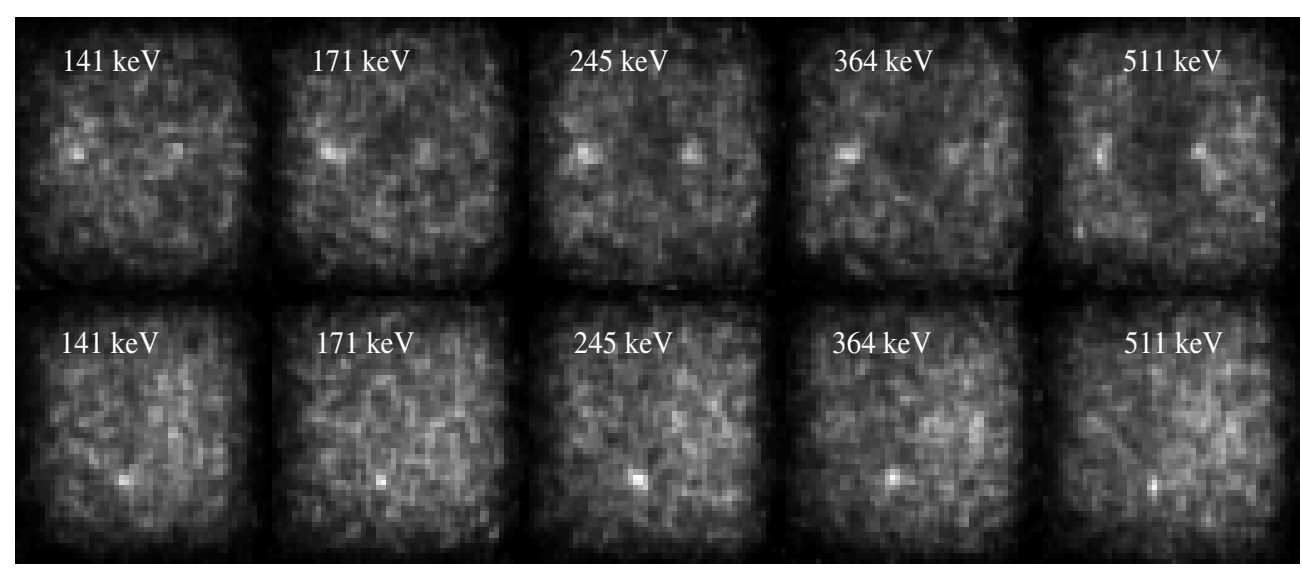

Figure 10. Penalized LMML-EM reconstruction of breast images with lead shielding (activity concentration ratio among breast, heart and liver is 1:2:4). Top row: medial plane. Bottom row: $1 \mathrm{~cm}$ below the medial plane. Two $8 \mathrm{~mm}$ tumours with T/B ratios of 8:1 and 10:1 are located in the medial plane. A $5 \mathrm{~mm}$ diameter tumour with a T/B ratio of $25: 1$ is located in the plane $1 \mathrm{~cm}$ below the medial plane.

shown for comparison. The contribution of liver counts to the right breast is $30 \%$ at $141 \mathrm{keV}$ and increases to $48 \%$ at $511 \mathrm{keV}$, about two times of that for the left breast. On the other hand, the left breast was obscured more than the right breast due to the counts from heart. The background from non-breast activity was drastically reduced by lead apron shielding. In particular, the counts from liver were less than $5 \%$ in both breasts at the energy of $141 \mathrm{keV}$. Figure 10 shows the craniocaudal views of the reconstructed images of right breast with the lead apron shielding at different energies. The imaging condition is the same as that in the lesion visibility study with an activity concentration ratio of 1:2:4 in breast, heart and liver. The effectiveness of the shielding reflected on the image quality compared to figure 8 is obvious, especially at low and medium energies. The $8 \mathrm{~mm}$ lesion obscured by background radiation at $141 \mathrm{keV}$ in figure 8 becomes visible with shielding. As gamma photon energy increases, thicker shielding is required.

\subsection{Performance comparison between collimated scintimammography and Compton camera based scintimammography}

Figure 11(a) shows the raw image of right breast at $141 \mathrm{keV}$ with the activity confined to the breast for the dedicated mechanically collimated scintimammographic system. The image size is $100 \times 100$ with a pixel size of $1 \mathrm{~mm}$. With the image acquisition time equivalent to that of the C-SMM system, the collimated scintimammographic system produces a much noisier, and considerably lower contrast image due to the poor sensitivity. All three tumours are barely visible while the same lesions are better visualized in the C-SMM system (figure 7). As can be seen, for the same imaging time, the C-SMM system performs better than the collimated system when there is no background contamination from the non-breast activity. The simulated parallel-hole collimated scintimammographic system (dual-head) has a counting efficiency of $2.7 \times 10^{-4}$ including tissue attenuation for a point source located at the geometric centre of a $5 \mathrm{~cm}$ thick breast phantom, about 110 times smaller than that of the C-SMM system. Figure 11(b) provides the corresponding smoothed image with a two-dimensional Gaussian filter. The $\sigma$ of the Gaussian filter is determined by the spatial resolution of the NaI detector 


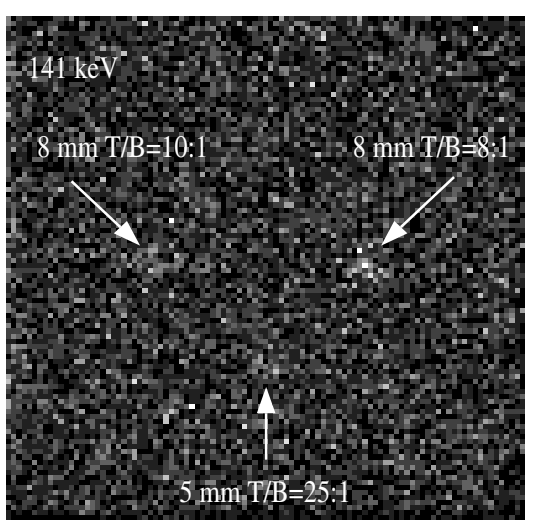

(a)

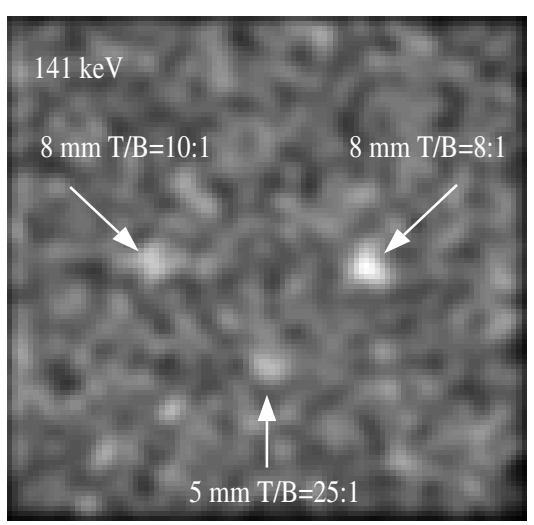

(b)

Figure 11. Planar image of breast with simulated lesions for the collimated system (only breasts and lesions contain activity). (a) Raw image. (b) Gaussian smoothed image.

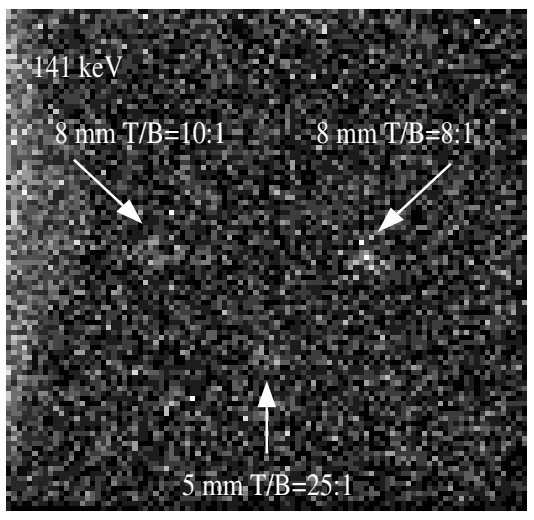

(a)

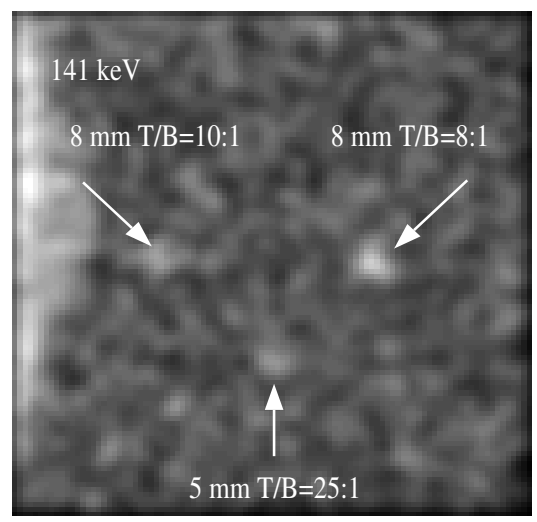

(b)

Figure 12. Planar image of breast with simulated lesions including heart and liver background for the collimated system (activity concentration ratio among breast, heart and liver is 1:3:12). (a) Raw image. (b) Gaussian smoothed image.

and the simulated parallel-hole collimator at the distance of $2.5 \mathrm{~cm}$, which is the depth of the mid-plane. The Gaussian kernel used in this study has a size of $5 \times 5$ and $\sigma$ of 1.77. The smoothed image has significantly reduced noise and all three lesions become visible. Figure 12(a) represents the raw image of right breast with the activity concentration ratio of 1:3:12 among normal breast, heart and liver. Again, the lesions are hardly seen. However, the image degradation due to background radiation is relatively small compared to the C-SMM system. Actually, for the compact, dedicated mechanically collimated scintimammographic system as shown in figure 4 , the scattering due to myocardium and liver activity is about 9.4\%. Figure 12(b) shows the smoothed image using the same Gaussian filter described previously. With the smoothing, all three simulated lesions are visualized. The reduced tumour-to-background contrast was observed in the $5 \mathrm{~mm}$ tumour and the $8 \mathrm{~mm}$ tumour close to the chest wall due to scattered counts from background activity in the heart and liver. Figure 13 shows the penalized LMML-EM reconstruction of breast images with the same activity concentration and imaging time for the shielded C-SMM system. A total of 


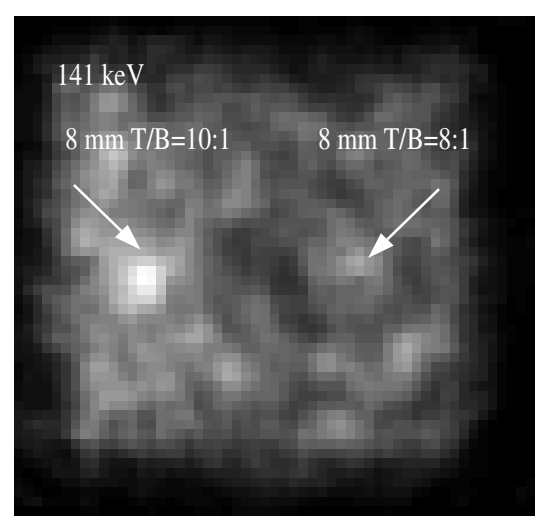

(a)

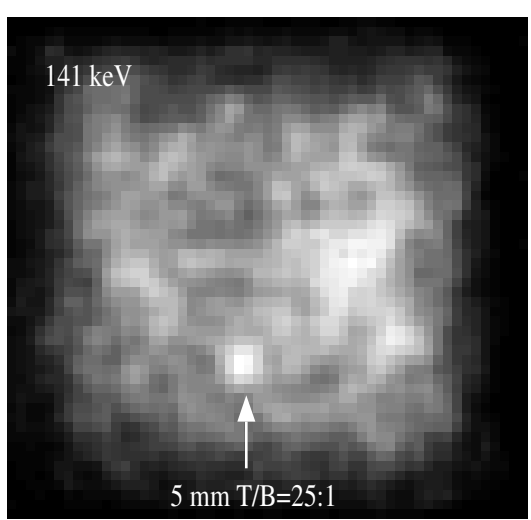

(b)

Figure 13. Penalized LMML-EM reconstruction of breast images including heart and liver background with lead shielding for the C-SMM system (activity concentration ratio among breast, heart and liver is 1:3:12). (a) Medial plane. (b) $1 \mathrm{~cm}$ below the medial plane.

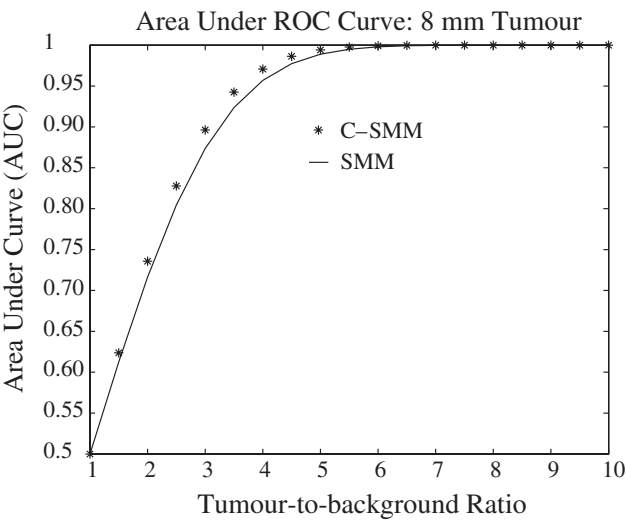

(a)

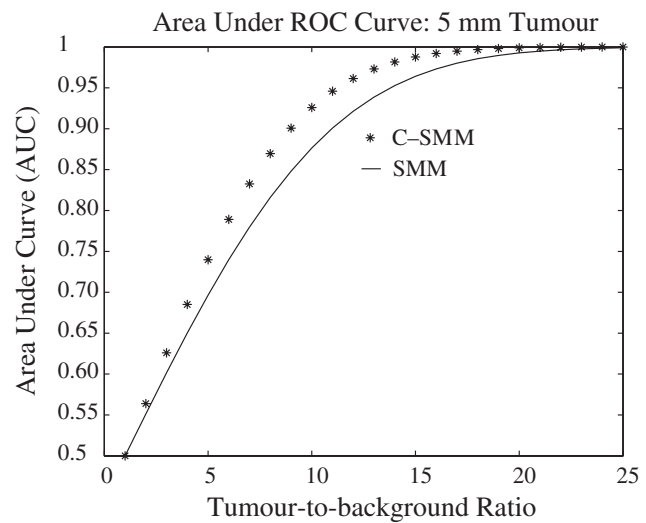

(b)

Figure 14. Area under the ROC curve for the task of detecting $8 \mathrm{~mm}$ and $5 \mathrm{~mm}$ diameter tumours with various $\mathrm{T} / \mathrm{B}$ ratios for ${ }^{99 \mathrm{~m}} \mathrm{Tc}$-sestamibi biodistribution for the collimated SMM and C-SMM systems. The same imaging time and activity concentration levels (breast:heart:liver $=1: 3: 12$ ) were used for both systems. The solid line represents AUC results for collimated SMM and asterisks indicate AUC results for C-SMM: (a) $8 \mathrm{~mm}$ tumour and (b) $5 \mathrm{~mm}$ tumour.

five slices in $1 \mathrm{~cm}$ intervals as shown in figure 2, were reconstructed with a pixel size of $2 \mathrm{~mm}$. Two slices through the centre of the tumours were displayed. The $8 \mathrm{~mm}$ lesion with a $\mathrm{T} / \mathrm{B}$ ratio of $8: 1$ is barely visible, the other two lesions can be clearly seen, however. The difference between this image and that in figure 10 at $141 \mathrm{keV}$ is the increased heart and liver background. Figures 14(a) and (b) show the results of the quantitative performance evaluation of the two systems in detecting an $8 \mathrm{~mm}$ and a $5 \mathrm{~mm}$ tumour with known $\mathrm{T} / \mathrm{B}$ ratios and a known background distribution. As can be seen, under the condition of equal imaging time, the C-SMM slightly outperforms the collimated SMM in detecting an $8 \mathrm{~mm}$ tumour close to the chest wall, especially in the range of $\mathrm{T} / \mathrm{B}$ ratios varying from $2.5: 1$ to $4.5: 1$. When the tumour size is reduced to $5 \mathrm{~mm}$, however, C-SMM outperforms collimated SMM by a moderate margin. In fact, a further improvement in lesion detectability could be expected for 
the proposed Compton camera based scintimammography if five slices rather than three were reconstructed as in section 2.3.3.

No significant difference was observed in the visual imaging quality of the two systems in lesion detection at low energy. The less favourable performance presented by the C-SMM system at $141 \mathrm{keV}$ is partially caused by the overestimated performance for the collimated system and the relative large decoding penalty associated with the Compton camera at this energy. However, quantitative evaluation using the NPWMF observer reveals the improved lesion detectability for the proposed scintimammographic system at $141 \mathrm{keV}$. A larger improvement over the dedicated SMM system was achieved in the detection of a $5 \mathrm{~mm}$ tumour. Although the proposed C-SMM system outperforms the dedicated SMM system in lesion detection by only a moderate amount, it demonstrates a very promising performance at higher energies. Furthermore, the C-SMM system can collect photons from a wide angular range, while only a single angle is sampled by the collimated system. This means that no tomographic information is available from the collimated system. Meanwhile, the C-SMM system achieves reasonable depth resolution even with limited-angle tomography. The C-SMM system can also image regions close to the chest wall, which is difficult to achieve with the collimated system.

\section{Conclusions}

In this paper, we propose an electronically collimated scintimammographic system based on the principle of the Compton-scatter camera. Since Compton cameras have much more favourable resolution-efficiency tradeoff in comparison with mechanical collimation, the proposed system substantially improves gamma-ray detection efficiency over conventional scintimammography while achieving good spatial resolution.

Using Monte Carlo simulations and a realistic anthropomorphic phantom, we have predicted the imaging performance of our system in terms of detection efficiency, spatial resolution and small lesion detection at different gamma photon energies. Our results demonstrate that this electronically collimated scintimammographic system can achieve a counting efficiency of 0.03 , more than two orders of magnitude improvement over that of a dedicated mechanically collimated system. At the same time, it has a FWHM spatial resolution of $3.8 \mathrm{~mm}$ for a point source located $2.5 \mathrm{~cm}$ deep in a $5 \mathrm{~cm}$ thick breast phantom at $141 \mathrm{keV}$. Through the lesion visibility experiments, we have shown that this system has high potential for detecting small tumours $(\leqslant 8 \mathrm{~mm})$, especially at high gamma photon energies. The non-prewhitening matched filter observer study reveals that the proposed system has an improved performance over the dedicated mechanically collimated scintimammographic system in detecting $8 \mathrm{~mm}$ and $5 \mathrm{~mm}$ tumours. However, the lesion contrast and visibility are greatly degraded due to substantial background from myocardium and liver. A simple lead apron shielding is shown to be effective in reducing the background by at least $50 \%$.

The advantages of the proposed C-SMM system are twofold. The high efficiency leads to increased count statistics for clinical scan durations at realistic levels of activity or greatly shortened imaging time with the same number of counts, which potentially improves the image quality by reducing the noise or image blurring due to patient movement. Furthermore, unlike the mechanically collimated system whose performance degrades significantly with the increased photon energy due to severe septal penetration, the performance of a Compton camera based system improves greatly at higher gamma photon energies. With the development of radiotracers labelled with higher energy gamma emitters applicable to breast imaging, the Compton camera based scintimammographic system is expected to outperform the conventional system by a large margin. The Compton imager is about 110 times more 
sensitive than a collimated system, but allowance must be made for the reduced information per detected photon due to conical ambiguity.

\section{Acknowledgments}

The authors would like to thank Dr Scott Wilderman for help and discussion on list-mode maximum likelihood expectation maximization reconstruction. This research was supported in part by the National Institute Health under grant nos R33CA88179-02 and EB000430-31.

\section{References}

Barrett H H, White T and Parra L C 1997 List-mode likelihood J. Opt. Soc. Am. 14 2914-23

Barrett H H, Yao J, Rolland J P and Myers K J 1993 Model observers for assessment of image quality Proc. Natl Acad. Sci. (Washington, DC, 13-14 Jan. 1992) pp 9758-65

Bielajew A F and Walker S 1993 Running EGS4 on different architectures The National Research Council of Canada Report PIRS-0392 (Ottawa: NRCC)

Blackman D K, Bennett E M and Miller D S 1999 Trends in self-reported use of mammograms (1989-1997) and Papanicolaou tests (1991-1997): behavioral risk factor surveillance system Morb. Mortal. Wkly. Rep. CDC Surveill. Summ. 48 1-22

Ciarmiello A et al 1998 Tumor clearance of technetium-99m-sestamibi as a predictor of response to neoadjuvant chemotherapy for locally advanced breast cancer J. Clin. Oncol. 16 1677-83

Cinti M N et al 2001 Tumor SNR analysis in scintimammography by dedicated high contrast image Proc. IEEE Nucl. Sci. Symp. Med. Imaging Conf. (San Diego, CA, 4-10 Nov. 2001) pp 1318-22

Cutrone J A et al 1999 Tc-99m sestamibi scintimammography for the evaluation of breast masses in patients with radiographically dense breasts Breast $J .5383-8$

Ernster V L et al 1996 Incidence of and treatment for ductal carcinoma in situ of the breast JAMA 275 913-8

ICRU 1989 Tissue substitutes in radiation dosimetry and measurement Report 44 of the International Commission on Radiation Units and Measurements (Bethesda, MD: ICRU)

ICRU 1996 Medical imaging-the assessment of image quality Report 54 of the International Commission on Radiation Units and Measurements (Bethesda, MD: ICRU)

Jackson V P, Hendrick R E, Feig S A and Kopans D B 1993 Imaging of the radiographically dense breast Radiology 188 297-301

Khalkhali J A et al 1995 Scintimammography: the complementary role of Tc-99m, sestamibi prone breast imaging for the diagnosis of breast carcinoma Radiology $196421-6$

Kim J H, Choi Y, Joo K S, Sihn B S, Chong J W, Kim S E, Lee K H, Choe Y S and Kim B T 2000 Development of a miniature scintillation camera using an $\mathrm{NaI}(\mathrm{Tl})$ scintillator and PSPMT for scintimammography Phys. Med. Biol. 45 3481-8

Levin C S, Hoffman E J, Tornai M P and MacDonald L R 1997 PSPMT and photodiode designs of a small scintillation camera for imaging malignant breast tumors IEEE Trans. Nucl. Sci. 44 1513-20

MacDonald L, Patt B E, Iwanczyk J S and Hoffman E J 2000 LumaGem: high resolution dedicated gamma camera J. Nucl. Med. $4156 \mathrm{P}$

Majewski S et al 2001 Optimization of dedicated scintimammography procedure using detector prototypes and compressible phantoms IEEE Trans. Nucl. Sci. 48 822-9

Marsaglia G, Zaman A and Tsang W W 1990 Toward a universal random number generator Stat. Probab. Lett. 8 35-9

Maublant J et al 1996 Technetium-99m-sestamibi uptake in breast tumor and associated lymph nodes J. Nucl. Med. 37 922-5

McElroy D P et al 2002 Evaluation of breast tumor detectability with two dedicated, compact scintillation cameras IEEE Trans. Nucl. Sci. 49 794-802

Meier D et al 2002 Silicon detector for a Compton camera in nuclear medical imaging IEEE Trans. Nucl. Sci. 49 812-6

Murthy K, Aznar M, Bergman A M, Thompson C J, Robar J L, Lisbona R, Loutfi A and Gagnon J H 2000 Positron emission mammographic instrument: initial results Radiology 215 280-5

Nuclear Fields 1999 Micro-cast collimators, vital for your imaging Product Description of Nuclear Fields

Orel S G, Schnall M D, Livolsi V A and Troupin R H 1994 MR imaging with radiologic-pathologic correlation Radiology 190 485-93 
Pandya S, Mackarem G, Lee A K C, McLellan R, Heatley G and Hughes K S 1998 Ductal carcinoma in situ: the impact of screening on clinical presentation and pathologic features Breast J. 4 146-51

Pani R, De Vincentis G, Scopinaro F, Pellegrini R, Soluri A, Weinberg I N, Pergola A, Scafe R and Trotta G 1998 Dedicated gamma camera for single photon emission mammography (SPEM) IEEE Trans. Nucl. Sci. 45 3127-33

Pani R et al 1996 Portable gamma camera for clinical use in nuclear medicine Proc. IEEE Nucl. Sci. Symp. Med. Imaging Conf. (Anaheim, CA, 2-9 Nov. 1996) pp 1170-4

Pani R, Scopinaro F, Pellegrini R, Soluri A, Weinberg I N and De Vincentis G 1997 The role of Compton background and breast compression on cancer detection in scintimammography Anticancer Res. 17 1645-9

Parra L C and Barrett H H 1998 EM algorithm and image quality estimation demonstrated on 2-D PET IEEE Trans. Med. Imaging 17 228-35

Pieper B C, Bowsher J E, Tornai M P, Peter J, Greer K and Jaszczak R J 2001 Breast tumor imaging using a tiltable head SPECT camera IEEE Trans. Nucl. Sci. 48 1477-82

Ries L A G et al 2003 SEER cancer statistics review, 1975-2000 National Cancer Institute (Bethesda, MD: NCI)

Rosenberg E D et al 1998 Effect of age, breast density, ethnicity, and estrogen replacement therapy on screening mammographic sensitivity and cancer stage at diagnosis: review of 183134 screening mammograms in Albuquerque, New Mexico Radiology 209 511-8

Savi A, Gerundini P, Zoli P, Maffioli L, Compierchio A, Colombo F, Matarrese M and Deutsch E 1989 Biodistribution of Tc-99m methoxy-isobutyl-isonitrile (MIBI) in humans Eur. J. Nucl. Med. 15 597-600

Studen A et al 2003 Development of silicon pad detectors and readout electronics for a Compton camera $\mathrm{Nucl}$. Instrum. Methods A $\mathbf{5 0 1} 273-9$

Thompson C J, Murthy K, Weinberg I N and Mako F 1994 Feasibility study for positron emission mammography Med. Phys. 21 529-38

Wackers F J Th et al 1989 Technetium-99m hexakis 2-methoxyisobutyl isonitrile: human biodistribution, dosimetry, safety, and preliminary comparison to thallium-201 for myocardial perfusion imaging J. Nucl. Med. 30 301-11

Wilderman S J, Fessler J A, Clinthorne N H, LeBlanc J W and Rogers W L 2001 Improved modeling of system response in list mode EM reconstruction of Compton scatter camera images IEEE Trans. Nucl. Sci. 48 111-6

Wilderman S J, Fessler J A, Clinthorne N H and Rogers W L 1998 List-mode maximum likelihood reconstruction of Compton scatter camera images in nuclear medicine Proc. IEEE Med. Imaging Conf. (Toronto, Canada, 1014 Nov. 1998) pp 1716-20

Williams M B, Goode A R, Reig V G, Majewski S, Weisenberger A G and Wojcik R 2000 Performance of a PSPMT based detector for scintimammography Phys. Med. Biol. 45 781-800

Wojcik R, Majewski S, Kross B, Steinbach D and Weisenberger A G 1998 High spatial resolution gamma imaging detector based on a 5" diameter R3292 Hamamatsu PSPMT IEEE Trans. Nucl. Sci. 45 487-91

Zhang L, Wilderman S J, Clinthorne N H and Rogers W L 2000 An anthropomorphic phantom integrated EGS4 Monte Carlo code and its application in Compton probe Proc. IEEE Nucl. Sci. Symp. Med.Imaging Conf. (Lyon, France, 10-15 Oct. 2000) pp 119-22

Zoglauer A 2000 Methods of image reconstruction for the MEGA Compton telescope (in German) Diploma Thesis Technical University Munich

Zubal I G and Harrell C R 1992 Voxel based Monte Carlo calculations of nuclear medicine images and applied variance reduction techniques Image Vis. Comput. 10 342-8 